\title{
More than 14 years' progress free survival of neratinib monotherapy for human epidermal growth factor receptor 2 (HER2) positive advanced breast cancer: a case report
}

\author{
Lina Zhang ${ }^{1}$, Shaohua Zhang ${ }^{2}$, Yang Yuan ${ }^{2}$, Jianbin Li $^{2}$, Feng Li ${ }^{2}$, Cuizhi Geng', Zefei Jiang ${ }^{2}$ \\ ${ }^{1}$ Department of Breast Center, The Fourth Hospital of Hebei Medical University, Shijiazhuang, China; ${ }^{2}$ Department of Breast Oncology, The Fifth \\ Medical Center of Chinese PLA General Hospital, Beijing, China \\ Correspondence to: Shaohua Zhang. Department of Breast Oncology, The Fifth Medical Center of Chinese PLA General Hospital, No. 8 East Street, \\ Fengtai District, Beijing 100071, China. Email: zhangsh19760428@163.com.
}

\begin{abstract}
Neratinib is an oral, irreversible, pan-tyrosine kinase inhibitor (TKI) of human epidermal growth factor receptor 1 (HER1), HER2, and HER4, which usually been used in combination with capecitabine for advanced HER2 positive breast cancer. Here we report an interesting case of a HER2 positive advanced breast cancer patient who received neratinib monotherapy with $240 \mathrm{mg}$ daily for more than 14 years after several lines of chemotherapy combined with trastuzumab and the target lesions in the liver nearly disappeared. As far as we know, this is the case with the longest benefit from neratinib monotherapy. A 56-year-old female was diagnosed HER2 positive breast cancer in 2004 and developed liver and bone metastases in 2006. Then she received several lines of chemotherapy including capecitabine, gemcitabine, and docetaxel combined with trastuzumab and the best curative effect was stable disease (SD). Then she was enrolled in a new drug (neratinib) clinical trial and treated with neratinib alone. The lesions in the liver were diminishing quickly and no longer growing up for more than 14 years. Due to the end of the clinical trial, the patient had to change drug to pyrotinib which had a similar mechanism with neratinib from August 2021. Patients may benefit from switching to another anti-HER2 drugs with different mechanism of action after the failure of prior anti-HER2 therapy.
\end{abstract}

Keywords: Human epidermal growth factor receptor-2 positive (HER2 positive); advanced breast cancer; neratinib; case report

Received: 30 September 2021; Accepted: 25 October 2021; Published: 31 October 2021.

doi: $10.21037 /$ tbcr-21-32

View this article at: https://dx.doi.org/10.21037/tbcr-21-32

\section{Introduction}

Human epidermal growth factor receptor-2 (HER2) expression accounts for approximately $20-30 \%$ of breast cancers and has been shown to associated with more aggressive tumors (1). Guidelines recommend anti-HER2 therapy throughout all the treatment periods of HER2 positive breast cancer, including neoadjuvant, adjuvant, and salvage therapy. Currently, anti-HER2 drugs used for breast cancer treatment mainly include macromolecule antibody drugs, antibody-drug conjugates (ADC) and tyrosine kinase inhibitors (TKI).
Neratinib is an oral, irreversible, pan-TKI of HER1, HER2, and HER4, which has been proved effective in patients with HER2-positive metastatic breast cancer $(2,3)$. Neratinib has been approved by the National Medical Products Administration (NMPA), European Medicines Agency (EMA) and the US Food and Drug Administration (FDA) for extended adjuvant treatment of early-stage, hormone receptor positive, HER2 positive breast cancer on the basis of the phase III ExteNET trial (4). And also FDA approved neratinib in combination with capecitabine for patients with advanced HER2 positive breast cancer after 2 prior lines of HER2-directed therapy on the basis of the 


\section{NALA trial (5).}

Although there is no indication for the use of neratinib monotherapy for advanced breast cancer, the case we report here who was enrolled in a Phase II clinical trial of HKI-272 (neratinib) 14 years ago and achieved longterm benefit from neratinib monotherapy. The progress free survival (PFS) of this patient was more than 14 years which may be the longest time with the treatment of neratinib. We present the following case in accordance with the CARE reporting checklist (available at https:// dx.doi.org/10.21037/tbcr-21-32).

\section{Case presentation}

A 56-year-old woman presented with a $3.0 \mathrm{~cm}$ diameter mass in her left breast and was diagnosed with breast cancer by core needle biopsy in Tianjin Cancer Hospital in July 2004. Imaging tests showed no distant metastases. Modified radical mastectomy for breast cancer was performed on 20July-2004. Postoperative histopathological examination revealed an invasive carcinoma with vascular invasion and the histological grade was grade II, with a size of $2.5 \mathrm{~cm}$ $\times 2.0 \mathrm{~cm}$ of the breast tumor. The axillary lymph nodes were $14 / 29$ positive. The pathological stage was T2N3M0. Immunohistochemistry evaluation showed that both the estrogen receptor (ER) and progesterone receptor (PR) were negative, and the HER2 was 2+ without further fluorescence in situ hybridization (FISH) test.

The patient was scheduled for adjuvant chemotherapy using paclitaxel $\left(210 \mathrm{mg}, 118 \mathrm{mg} / \mathrm{m}^{2}, \mathrm{~d} 1\right)$ combined with pirarubicin $\left(60 \mathrm{mg}, 34 \mathrm{mg} / \mathrm{m}^{2}\right.$, d1) every 3 weeks for 3 cycles followed by pirarubicin $\left(60 \mathrm{mg}, 34 \mathrm{mg} / \mathrm{m}^{2}\right.$, d1) combined with cyclophosphamide $\left(1,000 \mathrm{mg}, 562 \mathrm{mg} / \mathrm{m}^{2}, \mathrm{~d} 1\right)$ every 3 weeks for 3 cycles. The adjuvant chemotherapy began in August 2004 and ended in March 2005. And then the patient received adjuvant radiotherapy on the chest wall and the supraclavicular area (30 Gy/15 f).

The patient was diagnosed with liver and bone metastasis in April 2006 in Tianjin Cancer Hospital. The disease-free survival was 21 months. The patient was then given vinorelbine $\left(40 \mathrm{mg}, 22 \mathrm{mg} / \mathrm{m}^{2}, \mathrm{~d} 1\right)$ combined with carboplatin $\left(400 \mathrm{mg}, 220 \mathrm{mg} / \mathrm{m}^{2}, \mathrm{~d} 1\right)$ for 1 cycle, and the patient terminated the treatment due to the drug toxicity. Drug efficacy evaluation was not performed.

In September 2006, the patient came to the Fifth Medical Center of Chinese PLA General Hospital for further treatment. The FISH test was performed on the primary breast cancer tissue of the patient, and the

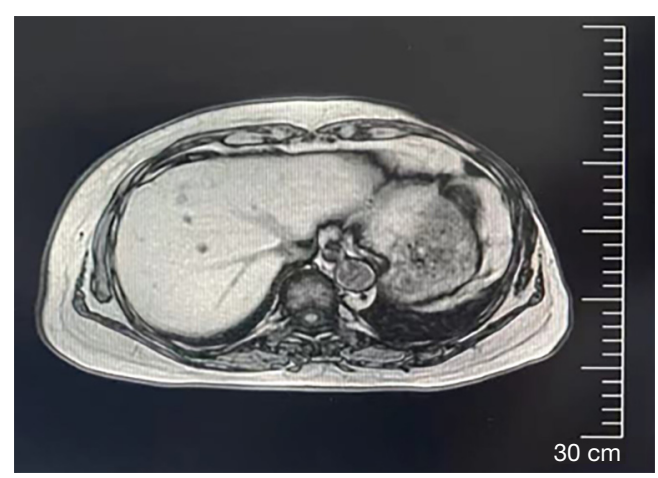

Figure 1 Liver MRI at the latest visit of the patient on August 2021. MRI, magnetic resonance imaging.

result showed HER2 amplification. And then the patient received capecitabine $\left(2,000 \mathrm{mg}, 1,136 \mathrm{mg} / \mathrm{m}^{2}\right.$, bid, $\left.\mathrm{d} 1-14\right)$ combined with trastuzumab $(8 \mathrm{mg} / \mathrm{kg}$ for the first time, and then $6 \mathrm{mg} / \mathrm{kg}$, d1) every 3 weeks for 6 cycles. The magnetic resonance imaging (MRI) showed the number and size of the lesions on the liver both increased. The assessment of the treatment was stable disease (SD) according to the Response Evaluation Criteria in Solid Tumor version 1.1 (RECIST 1.1) (6) retrospectively, and the time to progress (TTP) was 4 months. Then we changed capecitabine to gemcitabine $\left(1,800 \mathrm{mg}, 980 \mathrm{mg} / \mathrm{m}^{2}, \mathrm{~d} 1\right)$ combined with trastuzumab for only 2 cycles, the number and size of the lesions on the liver increased again. We changed the chemo drug for the second time. The patient received docetaxel (140 mg, $77 \mathrm{mg} / \mathrm{m}^{2}, \mathrm{~d} 1$ ) combined with trastuzumab every 3 weeks for 5 cycles, the treatment assessment showed SD. The last time of treatment was June $1^{\text {st }}, 2007$. The patient felt very fatigue due to the drug toxicity of docetaxel.

At this time, a new drug (HKI-272) clinical trial (protocol NO. B1891012) was recruiting subjects in the Fifth Medical Center of Chinese PLA General Hospital, and the patient was successfully screened. There were two target lesions in the liver with the size of $3.0 \mathrm{~cm} \times 2.7 \mathrm{~cm}$ and $3.0 \mathrm{~cm} \times 1.0 \mathrm{~cm}$ respectively at the baseline as recorded. But the images were unable to be obtained because of the image system update and the time elapsed. The patient began to receive HKI-272 (neratinib) $240 \mathrm{mg}$ per day from June 2007 until August 2021. One of the target lesions on the liver had disappeared, and the other lesion diminished to smaller than $0.5 \mathrm{~cm}$ (Figure 1). The assessment of the treatment was partial response (PR) (Figure 2). The most serious adverse event was grade II granulocytopenia for this patient. As we all know that diarrhea is one of the most common adverse 


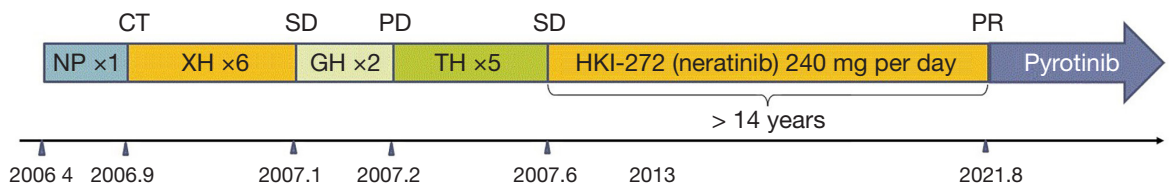

Figure 2 Timeline for the advanced breast cancer treatment of the patient. Treatment assessment: CT, cannot tolerate; SD, stable disease; PD, progression disease; PR, partial response; N, vinorelbine; P, carboplatin; X, capecitabine; H, trastuzumab; G, gemcitabine; T, docetaxel.

events, but for this patient, the most serious diarrhea grade was grade I and it only last for 3 months. And from about the $4^{\text {th }}$ month of the neratinib treatment, there was no diarrhea happened anymore. Unfortunately, due to the end of the clinical trial, the sponsor no longer provided free research drug (neratinib), and neratinib was not available in China, so the follow-up treatment of the patient was changed to pyrotinib which has a similar mechanism of action with neratinib.

In view of more than 14 years' benefit of neratinib monotherapy, we collected the patient's peripheral blood for next-generation sequencing (NGS), in order to find the potential biomarkers related to the benefit of neratinib after obtaining the informed consent of the patient. The results showed no clinically significant mutations in this patient.

All procedures performed in studies involving human participants were in accordance with the ethical standards of the institutional and/or national research committee(s) and with the Helsinki Declaration (as revised in 2013). Written informed consent was obtained from the patient for publication of this case report and accompanying images. A copy of the written consent is available for review by the editorial office of this journal.

\section{Discussion}

As a pan-TKI, neratinib in combination with chemotherapy had shown initial efficacy in advanced HER2 positive breast cancer (2,3). In the NALA (5) study, the median PFS of neratinib combined with capecitabine was 8.8 months among patients with advanced HER2 positive breast cancer treated with more than 2 lines of prior anti-HER2 therapy. Compared to lapatinib combined with capecitabine, neratinib combined with capecitabine increased the time to central nervous system (CNS) metastasis. It was based on this study that the FDA approved neratinib for the treatment of advanced HER2 positive breast cancer who had received at least two lines of prior anti-HER2 therapies.

In early-stage breast cancer, neratinib monotherapy has been approved by NMPA, FDA, and EMA for the intensive treatment of HER2 positive breast cancer after adjuvant therapy with trastuzumab-based on the study of ExteNET (4). Although neratinib monotherapy has not been approved for recommendation in advanced breast cancer, a phase II open-label clinical trial (7) showed the median PFS for neratinib monotherapy in patients with HER2 positive breast cancer who had been previously treated with trastuzumab was 22.3 weeks. The patient in this report received neratinib monotherapy after several lines of chemotherapy and trastuzumab and the lesions in the liver had almost disappeared. The PFS lasted more than 14 years, much longer than the 22.5 weeks in the phase II study.

The main adverse event of neratinib is diarrhea, mainly because neratinib can inhibit HER1, which causes chloride ion transport disorder leading to secretory diarrhea (8). The incidence of grade III diarrhea was as high as $30 \%$ in ExteNET study (4), and the diarrhea was a self-limiting disease. The highest incidence occurred during the first cycle of treatment and decreased progressively throughout the subsequent treatment. The control study (9) showed that prophylactic use of antidiarrheal drugs can reduce the incidence of grade 3 diarrhea caused by neratinib. This patient had grade I diarrhea mainly in the first 3 months of neratinib treatment, and in the following cycle, the diarrhea did not occur anymore. The patients were treated with montmorillonite powder for $3 \mathrm{~g}$ twice a day at the beginning of the application of neratinib. The patient's stool was 1-2 times more than usual a day. After 14 days, the patient stopped the treatment of montmorillonite powder because she felt that this degree of diarrhea did not affect her daily life and the diarrhea didn't happen anymore after 3 months from the beginning of the treatment of neratinib.

The patient felt delighted and lucky that she had been receiving neratinib therapy for more than 14 years and the lesions on her liver nearly disappeared. Due to the termination of the clinical trial, the patient could not continue the treatment of neratinib. Pyrotinib is another 
irreversible TKI that has a similar mechanism to neratinib. In view of the results of PHENIX (10) and PHOEBE (11) studies, pyrotinib had been approved by NMPA for the treatment of advanced HER2 positive breast cancer. The patient began treatment with pyrotinib in August 2021.

Specific gene mutations are often associated with mechanisms of drug resistance. And also, some specific gene mutations can help us select drugs that have potential efficacy. Cocco's study showed that HER2 mutations were present in $~ 7 \%$ of HER2-positive tumors which may be associated with resistance to trastuzumab and lapatinib but sensitivity to neratinib (12). As for this patient, there were no HER2 mutations detected but she remained sensitive to neratinib. This case is an exceptional example of the longterm benefit of neratinib, and not all patients with HER2 positive breast cancer will benefit from neratinib treatment. Therefore, there is still a lot of work to do for us to screen patients who were sensitive to a specific medicine.

\section{Conclusions}

Here we reported a patient with HER2 positive advanced breast cancer who received neratinib monotherapy after several lines of chemotherapy combined with trastuzumab and the PFS was more than 14 years. From this case, we found that some patients may be very sensitive to TKI and are not readily developing resistance to the drug. NGS on more similar cases may help to screen out such patients.

\section{Acknowledgments}

The authors are grateful to the patient, clinicians, pathologists and statisticians who participated in this study. Funding: None.

\section{Footnote}

Reporting Checklist: The authors have completed the CARE reporting checklist. Available at https://dx.doi. org/10.21037/tbcr-21-32

Conflicts of Interest: All authors have completed the ICMJE uniform disclosure form (available at https://dx.doi. org/10.21037/tbcr-21-32). JL serves as an unpaid Managing Editor of Translational Breast Cancer Research. CG serves as an unpaid editorial board member of Translational Breast Cancer Research. ZJ serves as an unpaid Editor-in-Chief of Translational Breast Cancer Research. The other authors have no conflicts of interest to declare.

Ethical Statement: The authors are accountable for all aspects of the work in ensuring that questions related to the accuracy or integrity of any part of the work are appropriately investigated and resolved. All procedures performed in studies involving human participants were in accordance with the ethical standards of the institutional and/or national research committee(s) and with the Helsinki Declaration (as revised in 2013). Written informed consent was obtained from the patient for publication of this case report and accompanying images. A copy of the written consent is available for review by the editorial office of this journal.

Open Access Statement: This is an Open Access article distributed in accordance with the Creative Commons Attribution-NonCommercial-NoDerivs 4.0 International License (CC BY-NC-ND 4.0), which permits the noncommercial replication and distribution of the article with the strict proviso that no changes or edits are made and the original work is properly cited (including links to both the formal publication through the relevant DOI and the license). See: https://creativecommons.org/licenses/by-nc-nd/4.0/.

\section{References}

1. Slamon DJ, Clark GM, Wong SG, et al. Human breast cancer: correlation of relapse and survival with amplification of the HER-2/neu oncogene. Science 1987;235:177-82.

2. Saura C, Garcia-Saenz JA, Xu B, et al. Safety and efficacy of neratinib in combination with capecitabine in patients with metastatic human epidermal growth factor receptor 2-positive breast cancer. J Clin Oncol 2014;32:3626-33.

3. Freedman RA, Gelman RS, Anders CK, et al. TBCRC 022: A Phase II Trial of Neratinib and Capecitabine for Patients With Human Epidermal Growth Factor Receptor 2-Positive Breast Cancer and Brain Metastases. J Clin Oncol 2019;37:1081-9.

4. Martin M, Holmes FA, Ejlertsen B, et al. Neratinib after trastuzumab-based adjuvant therapy in HER2-positive breast cancer (ExteNET): 5-year analysis of a randomised, double-blind, placebo-controlled, phase 3 trial. Lancet Oncol 2017;18:1688-700.

5. Saura C, Oliveira M, Feng YH, et al. Neratinib Plus Capecitabine Versus Lapatinib Plus Capecitabine in HER2-Positive Metastatic Breast Cancer Previously 
Treated With $\geq 2$ HER2-Directed Regimens: Phase III NALA Trial. J Clin Oncol 2020;38:3138-49.

6. Eisenhauer EA, Therasse P, Bogaerts J, et al. New response evaluation criteria in solid tumours: revised RECIST guideline (version 1.1). Eur J Cancer 2009;45:228-47.

7. Burstein HJ, Sun Y, Dirix LY, et al. Neratinib, an irreversible ErbB receptor tyrosine kinase inhibitor, in patients with advanced ErbB2-positive breast cancer. J Clin Oncol 2010;28:1301-7.

8. Andreyev J, Ross P, Donnellan C, et al. Guidance on the management of diarrhoea during cancer chemotherapy. Lancet Oncol 2014; 15:e447-60.

9. Barcenas CH, Hurvitz SA, Di Palma JA, et al. Improved tolerability of neratinib in patients with HER2-positive early-stage breast cancer: the CONTROL trial. Ann

doi: $10.21037 /$ tbcr-21-32

Cite this article as: Zhang L, Zhang S, Yuan Y, Li J, Li F, Geng C, Jiang Z. More than 14 years' progress free survival of neratinib monotherapy for human epidermal growth factor receptor 2 (HER2) positive advanced breast cancer: a case report. Transl Breast Cancer Res 2021;2:32.
Oncol 2020;31:1223-30.

10. Ma F, Ouyang Q, Li W, et al. Pyrotinib or Lapatinib Combined With Capecitabine in HER2-Positive Metastatic Breast Cancer With Prior Taxanes, Anthracyclines, and/or Trastuzumab: A Randomized, Phase II Study. J Clin Oncol 2019;37:2610-9.

11. Xu B, Yan M, Ma F, et al. Pyrotinib plus capecitabine versus lapatinib plus capecitabine for the treatment of HER2-positive metastatic breast cancer (PHOEBE): a multicentre, open-label, randomised, controlled, phase 3 trial. Lancet Oncol 2021;22:351-60.

12. Cocco E, Javier Carmona F, Razavi P, et al. Neratinib is effective in breast tumors bearing both amplification and mutation of ERBB2 (HER2). Sci Signal 2018;11:aat9773. 